\title{
Adult right-sided Morgagni hernia with Chilaiditi syndrome: A case report
}

\author{
Mustafa Korkut ${ }^{1} \oplus$, Sefa Türkoğlu² ${ }^{2}$ \\ ${ }^{1}$ Department of Emergency Medicine, Health Science University, Antalya Training and Research Hospital, Antalya, Turkey \\ ${ }^{2}$ Department of Radiology, Denizli State Hospital, Denizli, Turkey
}

\begin{abstract}
Chilaiditi syndrome, which is the symptomatic interposition of the bowel under the right hemidiaphragm, is a rare condition that can usually be managed without surgery. Morgagni hernia is an unusual disease that accounts for approximately $3 \%$ of congenital diaphragmatic hernias. A 66-year-old female presented to the emergency department with abdominal pain, nausea, and constipation. Computed tomography findings were consistent with the diagnosis of Morgagni hernia and Chilaiditi syndrome. In this article, we present this case due to the rare coexistence of these two conditions and the typical radiological findings.
\end{abstract}

Keywords: Chilaiditi syndrome, diaphragmatic hernia, Morgagni hernia.

Morgagni hernia (MH) is an uncommon condition characterized by congenital herniation through a retrosternal defect in the diaphragm. Although it is usually found incidentally, it may also occur with intestinal obstruction or ischemia. Usually observed in children, adults only comprise a minority of cases. ${ }^{[1,2]}$ Chilaiditi sign is a rare radiographic finding of subdiaphragmatic radiolucency that occurs with the interposition of the intestines between the liver and the diaphragm and is called Chilaiditi syndrome (CS) when associated with digestive symptoms..$^{[3]}$ This case report presents a patient who was admitted to the emergency department with complaints of abdominal pain and was diagnosed with $\mathrm{MH}$ associated with CS.

\section{CASE REPORT}

A 66-year-old female presented to the emergency department with upper abdominal pain, nausea, and anorexia lasting for two days.
She denied chronic constipation and her last bowel movement was two days earlier. Physical examination revealed a moderately distended abdomen with tenderness in the right upper quadrant and slightly decreased bowel sounds with no guarding or rebound tenderness. Her past medical history was unremarkable. On admission, her temperature was $37.4^{\circ} \mathrm{C}$, her heart rate was 88 beats/min, her respiratory rate was 16 breaths/min, and her blood pressure was $110 / 70 \mathrm{mmHg}$. Apart from minimal leukocytosis, the patient's laboratory values were normal.

Abdominal X-ray showed small-bowel gas and computed tomography $(\mathrm{CT})$ revealed the transverse colon located above the right lobe of the liver, known as Chilaiditi's sign. A small region of intestinal fat tissue was also seen in the loweranterior of the right thoracic cavity, probably due to the congenital hernia (Figure 1,2). With these findings, the patient was diagnosed as right $\mathrm{MH}$ and CS. The patient was hospitalized in the

Received: September 20, 2019 Accepted: October 11, 2019 Published online: April 15, 2020

Correspondence: Mustafa Korkut, MD. SBÜ Antalya Eğitim ve Araştırma Hastanesi Acil Tıp Kliniği, 07100 Muratpaşa, Antalya, Türkiye. Tel: +90 507 - 9719375 e-mail: drmustafakorkut@gmail.com 


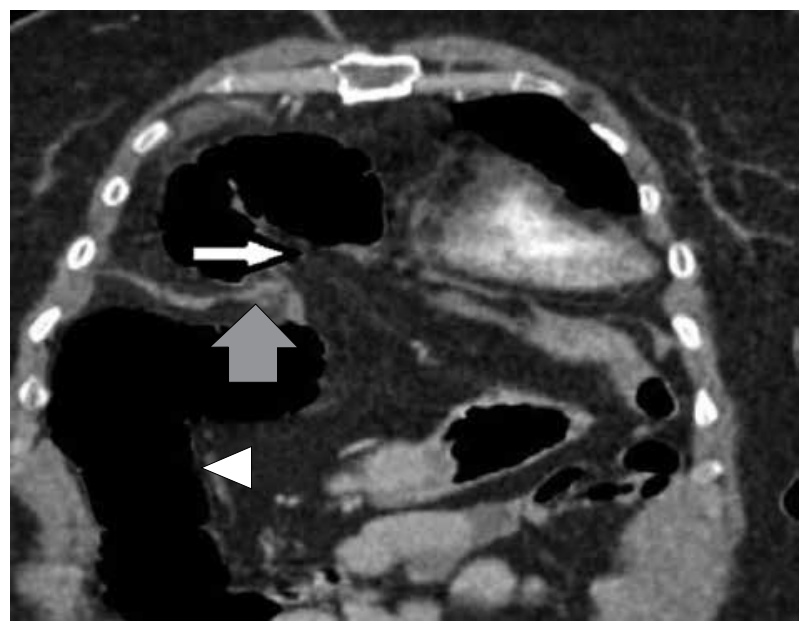

Figure 1. Coronal computed tomography image showing multiple bowel loops within the right thoracic cavity (white arrow) consistent with a Morgagni hernia. The diaphragm can be identified below the hernia contents (gray arrow) and the transverse colon located above the right lobe of the liver (arrowhead).

general surgery ward. She received intravenous fluids, oral laxatives, and water enemas. After numerous bowel movements, the patient's pain and distention resolved. Elective surgery was planned and the patient was discharged on the fourth day of hospitalization with oral laxatives and enemas.

\section{DISCUSSION}

Chilaiditi syndrome is a rare radiological finding of segmental interposition of the colon between the liver and diaphragm. The syndrome was first defined in 1911 by the Greek radiologist Demetrious Chilaiditi, who described three cases of the radiographic anomaly. ${ }^{[4]}$ Conversely, radiographic findings without any clinical symptoms are called Chilaiditi signs. The prevalence of the disease is thought to range between $0.025 \%$ and $0.28 \% .^{[5,6]}$ Chilaiditi syndrome is generally asymptomatic and usually diagnosed incidentally. Its most common presentation is abdominal pain, nausea, vomiting, distention, changes in bowel habits, chest pain, shortness of breath, and respiratory distress. ${ }^{[4,5]}$ In our case, the patient presented with CS-like radiological findings.

Morgagni hernia was first described in 1769 as a herniation of the intraabdominal organs into the thorax through a parasternal or retrosternal

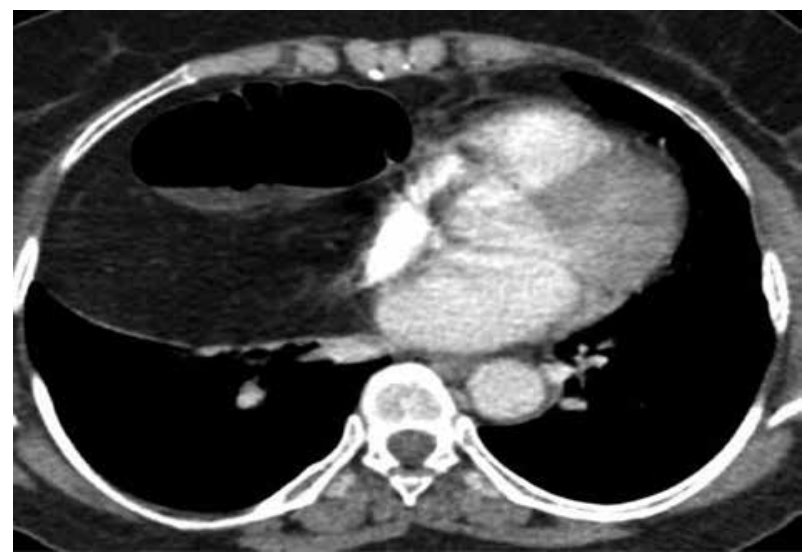

Figure 2. Axial computed tomography image showing hernia intestinal fat tissue in the right thoracic cavity and minimally enlarged colonic diameter.

defect of the diaphragm. ${ }^{[7]}$ The hernia usually appears on the right side, but can also occur on the left side or midline. These are the rarest of congenital diaphragmatic hernias, accounting for only 2-3\% of cases. ${ }^{[8]}$ Morgagni hernia is generally predominant in females and is associated with other anomalies such as ventricular septal defect, dextrocardia, Down's syndrome, Turner's syndrome, Noonan's syndrome, Prader Willi syndrome, tetralogy of Fallot, scoliosis, and omphalocele. ${ }^{[7]}$ Most $\mathrm{MH}$ is diagnosed late because patients may be asymptomatic or present with ambiguous gastrointestinal and respiratory symptoms and signs. ${ }^{[2]}$ The clinical presentation of this patient was simply misleading with the delayed diagnosis of pulmonary symptoms. In chest radiography, $\mathrm{MH}$ is often randomly diagnosed as homogeneous masses in the right cardiophrenic angle. In case of involvement of a hollow viscus, an air fluid level may be recorded in the thoracic cavity. ${ }^{[2,8]}$ Although ultrasonography has been shown to be useful in assessing diaphragmatic hernias, CT is the most sensitive imaging method because it provides excellent anatomical detail of complications such as strangulation. ${ }^{[9]}$ In our case, the patient presented with similar complaints including abdominal pain, nausea, and anorexia.

The treatment of CS varies depending on the severity of the symptoms. Asymptomatic patients do not require any intervention and patients with mild or intermittent symptoms can usually be managed with conservative treatment. ${ }^{[5,6]}$ Surgical treatment is reserved for patients whose symptoms 
do not resolve or patients with complications such as ischemia or perforation. ${ }^{[10]}$ Once diagnosed, the need for surgery largely depends on presentation. Although the majority of these hernias are asymptomatic, repair is recommended to prevent future complications. Emergency intervention is not always necessary unless there is evidence of strangulation. ${ }^{[8,11]}$ In this case, the patient's symptoms completely resolved with conservative treatment.

In conclusion, a highly rare case of a right-sided $\mathrm{MH}$ associated with $\mathrm{CS}$ in an adult who resolved with conservative treatment was described. Chilaiditi syndrome and $\mathrm{MH}$ are usually asymptomatic and the disorder may have late manifestation. Symptoms and clinical condition may vary from mild to severe, therefore clinicians should consider these disorders in their daily exams.

\section{Declaration of conflicting interests}

The authors declared no conflicts of interest with respect to the authorship and/or publication of this article.

\section{Funding}

The authors received no financial support for the research and/or authorship of this article.

\section{REFERENCES}

1. Swain JM, Klaus A, Achem SR, Hinder RA. Congenital diaphragmatic hernia in adults. Semin Laparosc Surg
2001:8:246-55.

2. Eren S, Gümüș H, Okur A. A rare cause of intestinal obstruction in the adult: Morgagni's hernia. Hernia 2003;7:97-9.

3. Aldoss IT, Abuzetun JY, Nusair M, Suker M, Porter J. Chilaiditi syndrome complicated by cecal perforation. South Med J 2009;102:841-3.

4. Takahashi K, Ito H, Katsube T, Tsuboi A, Hashimoto $\mathrm{M}$, Ota $\mathrm{E}$, et al. Treatment of Chilaiditi syndrome using laparoscopic surgery. Asian J Endosc Surg 2017;10:63-5.

5. Moaven O, Hodin RA. Chilaiditi syndrome: a rare entity with important differential diagnoses. Gastroenterol Hepatol (N Y) 2012;8:276-8.

6. Kang D, Pan AS, Lopez MA, Buicko JL, Lopez-Viego M. Acute abdominal pain secondary to chilaiditi syndrome. Case Rep Surg 2013;2013:756590.

7. Nasr A, Fecteau A. Foramen of Morgagni hernia: presentation and treatment. Thorac Surg Clin 2009;19:463-8.

8. Arora S, Haji A, Ng P. Adult Morgagni hernia: the need for clinical awareness, early diagnosis and prompt surgical intervention. Ann R Coll Surg Engl 2008;90:694-5.

9. Colakoğlu O, Haciyanli M, Soytürk M, Colakoğlu G, Simşek I. Morgagni hernia in an adult: atypical presentation and diagnostic difficulties. Turk $\mathrm{J}$ Gastroenterol 2005;16:114-6.

10. Kapania EM, Link C, Eberhardt JM. Chilaiditi Syndrome: A Case Report Highlighting the Intermittent Nature of the Disease. Case Rep Med 2018;2018:3515370.

11. Bedel, C, Coşkun, G. A rare cause of abdominal pain: Portal vein thrombosis. İstanbul Bilim Üniversitesi Florence Nightingale Tip Dergisi 2018;4:102-5. 